\title{
Comportement des métaux à l'usine marémotrice de la Rance
}

\author{
par M. Leboróne \\ Service de la Production Hydraulique \\ E.D.F., Brive
}

Après l'exposé général que vient de faire M. Faral mentionnant, entre autres, les différents métaux utilisés dans les groupes et vannes de l'usine marémotrice de la Rance, nous allons examiner maintenant le comportement «corrosion» de ces métaux en exploitation.

Nous devons, au préalable, signaler l'existence d'une difficulté dans l'analyse de ce comportement; en effet, l'utilisation d'une protection cathodique, remarquablement efficace, ne permet pas toujours de savoir si le comportement satisfaisant de tel métal noble le serait également sans cette protection; cette difficulté est toutefois atténuée par le fait que les phénomènes de corrosion, en eau de mer, sont extrêmement intenses et qu'il suffit d'une immersion de durée relativement faible, sans protection, pour déjà avoir une idée du comportement du métal.

\section{2}

Le tableau 1 rappelle brièvement, pour chaque catégorie de métaux nobles, les principales pièces constitutives des groupes, vannes et portes d'écluses de l'usine marémotrice de la Rance.

Nous allons examiner successivement le comportement des pièces constitutives des groupes bulbes, des vannes de barrage, des écluses et enfin des tuyauteries.

Il est indispensable de procéder ainsi, car les conditions d'immersion de ces différents matériels n'ont pas été les mêmes, notamment en ce qui concerne la mise en service de la protection cathodique.

\section{3. - Comportement des métaux des groupes bulbes}

Dans l'ensemble, la tenue à la corrosion des pièces métalliques est excellente, grâce, d'ailleurs, à la protection cathodique dont MM. Legrand et Lambert vous parleront.

Heureusement pour les scientifiques, et malheureusement pour les exploitants, il y a eu pour deux groupes bulbes, deux périodes d'immersions différentes, permettant de connaître l'effet de la corrosion avec et sans protection cathodique.

- une première période où les deux groupes sont restés en eau stagnante, donc à l'arrêt, pendant six mois dont deux sans protection cathodique;

- une deuxième période, suivant la précédente, où les groupes étaient en service normal avec la protection cathodique.

3.1. Pendant la première période d'immersion, sans rotation et sans protection cathodique,

il s'est développé à la surface des pièces en acier, non protégées par la peinture et la couche d'antifouling, c'est-àdire essentiellement les pales en acier martensitique $17 / 4$, de très importants dépôts d'algues et de coquillages. En dessous de ce dépôt, on a découvert de très nombreux points d'attaques sous formes de petits cratères, ayant nécessité une réparation par soudure. Ces attaques sont dues à une corrosion par aération différentielle. Les figures 1 à 6 montrent l'état des pales après les cinq mois d'immersion. 


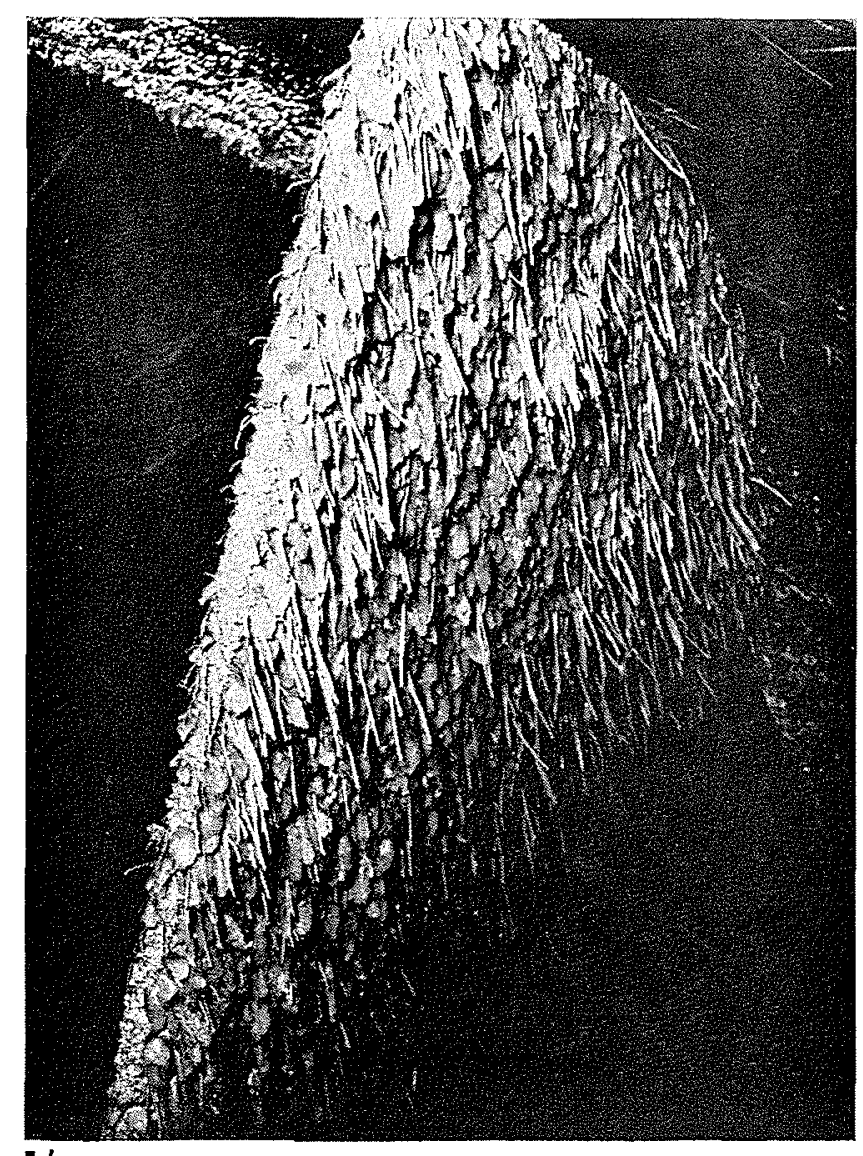

II

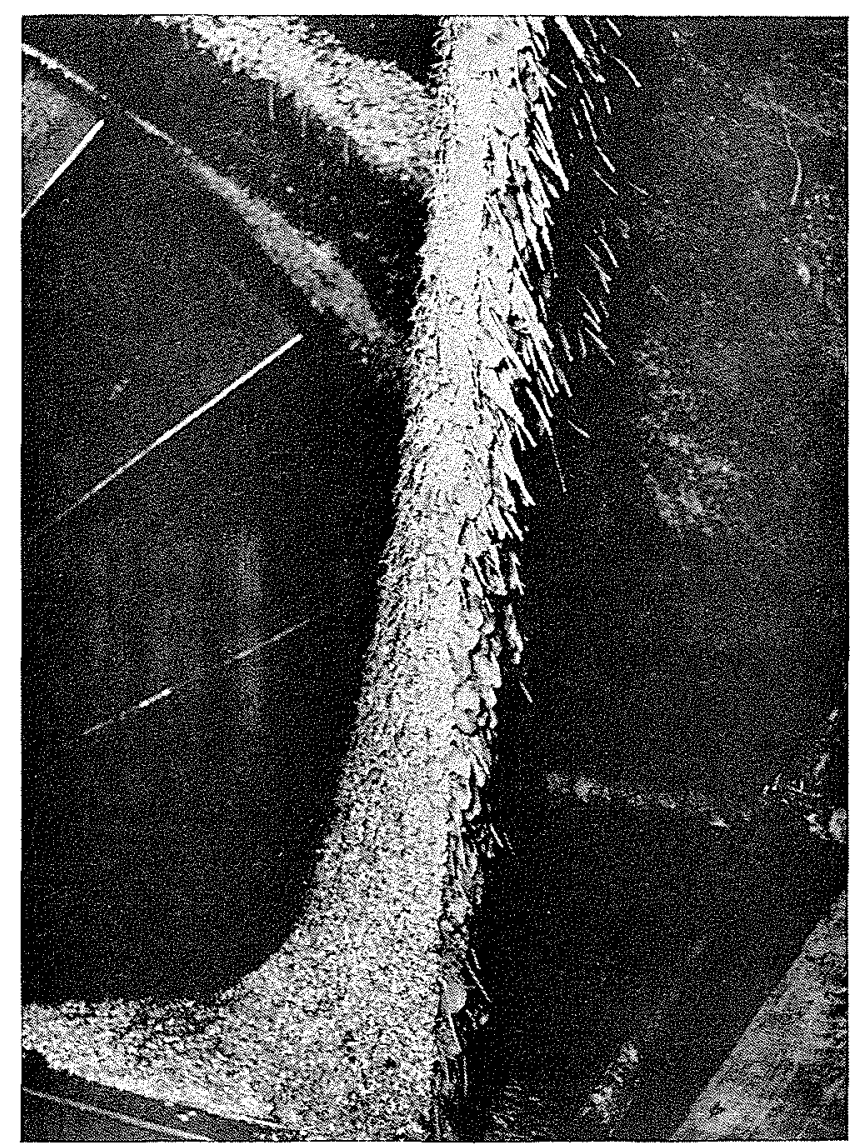

2)

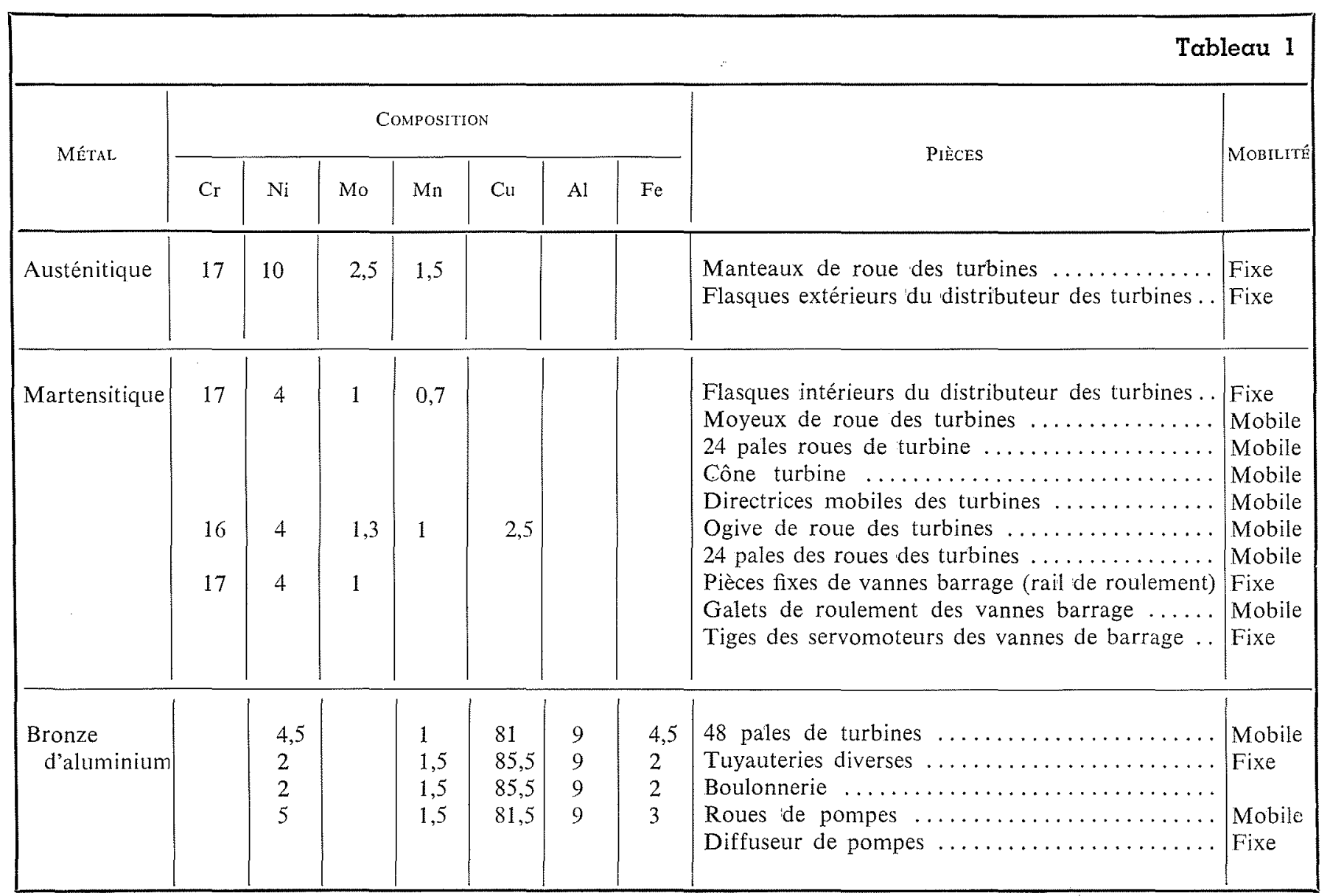




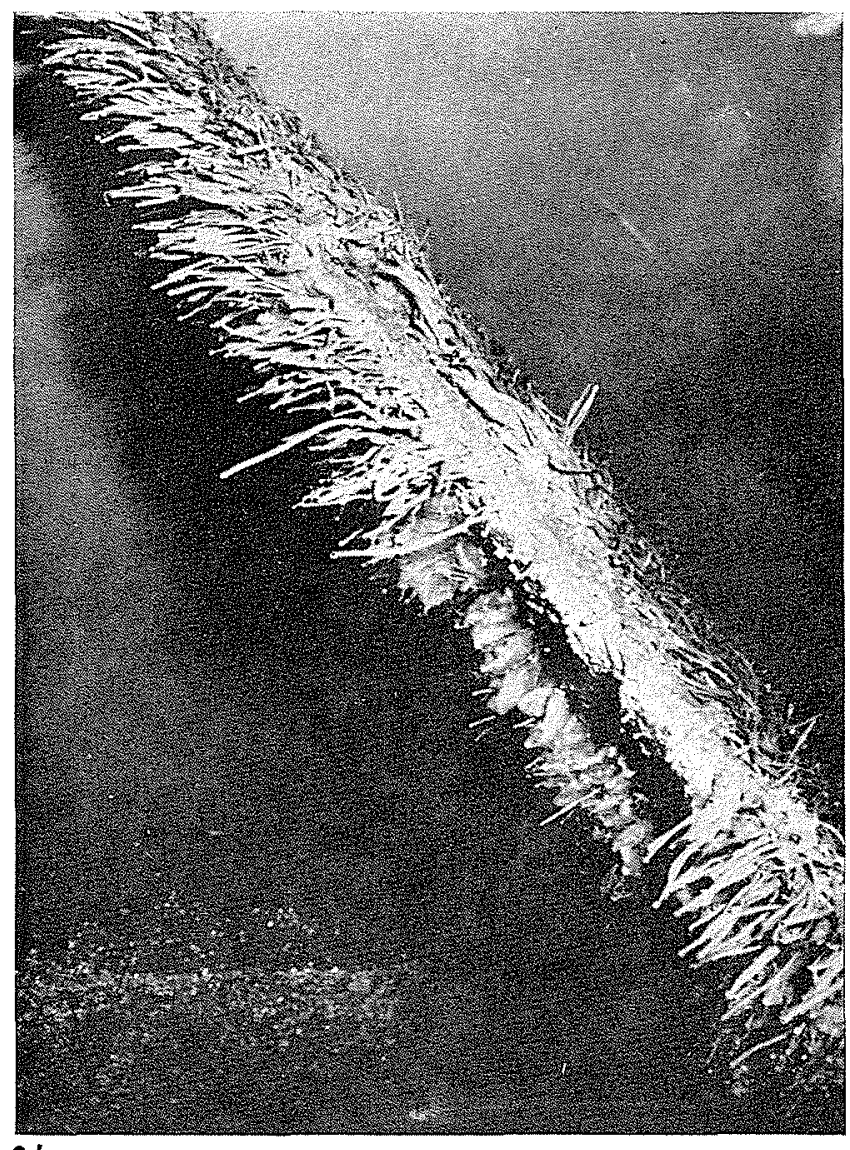

3/

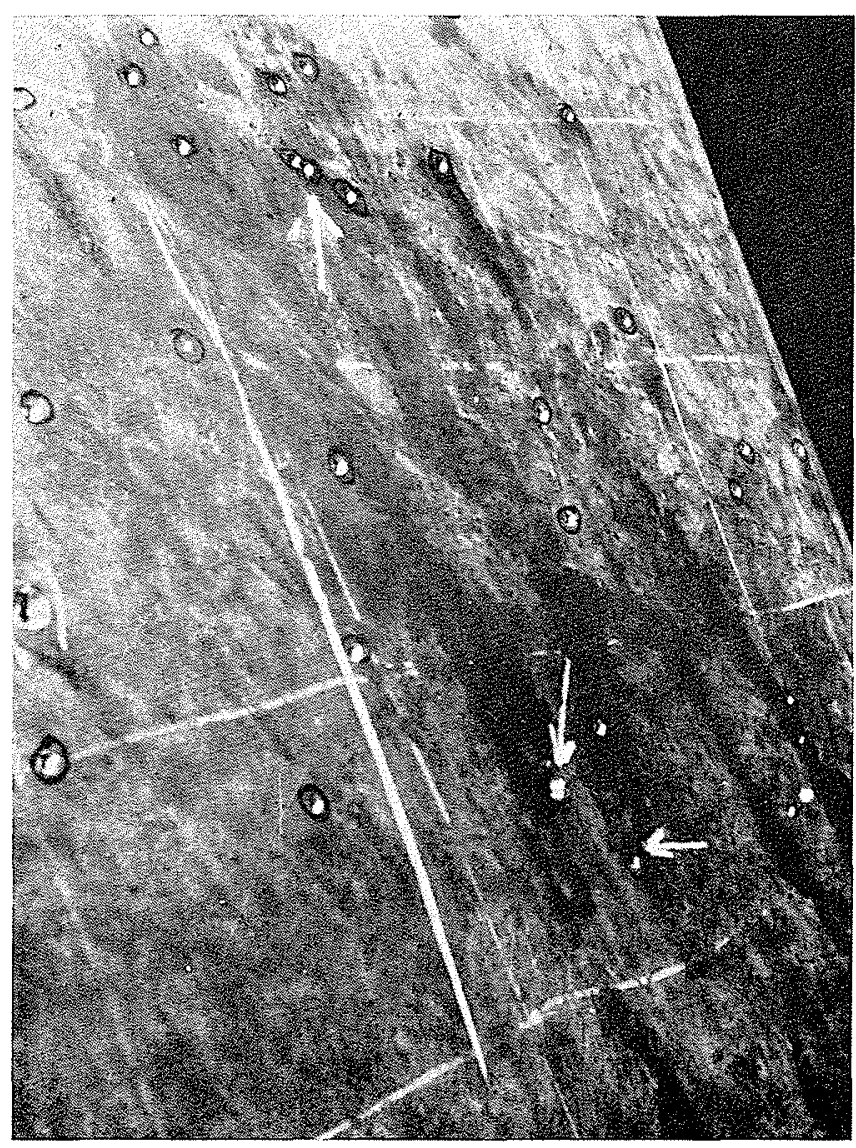

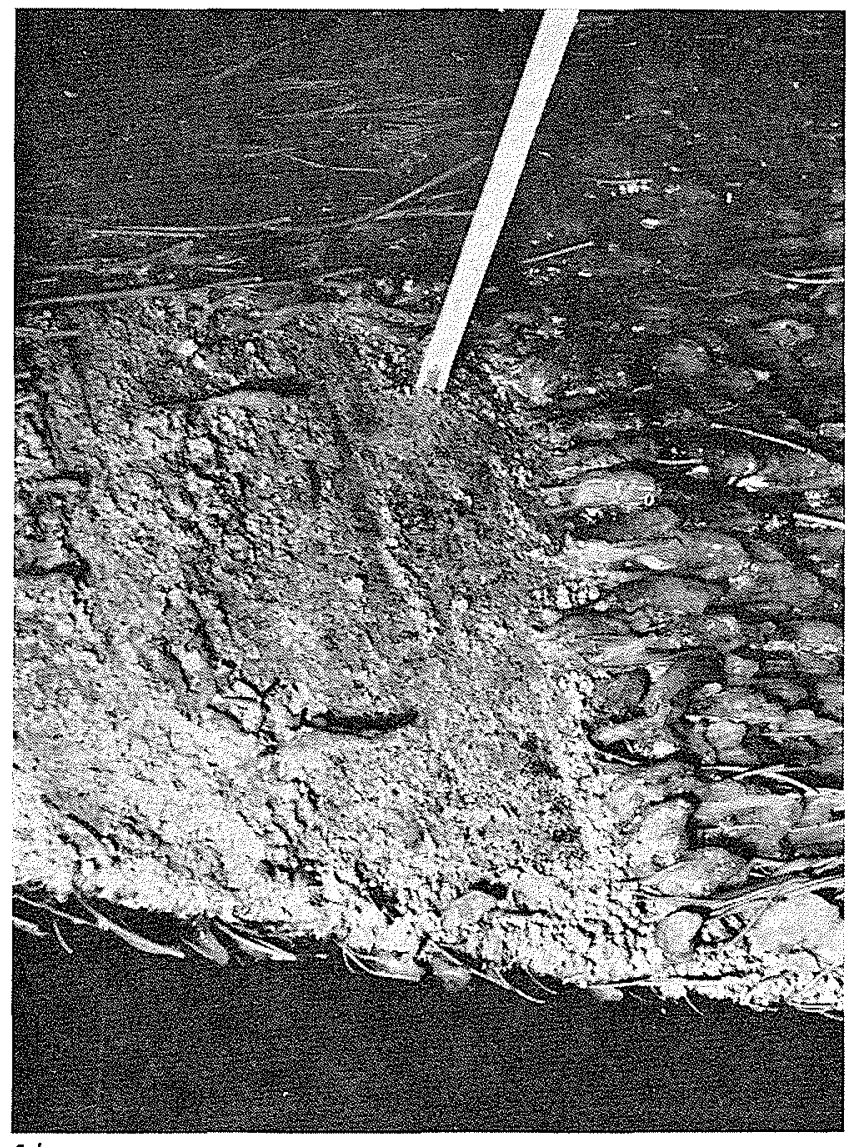

4/

1 à $6 /$

Etat des pales du groupe B 6 à l'usine marémotrice après cinq mois d'immobilisation en eau de mer, dont deux sans protection cathodique.

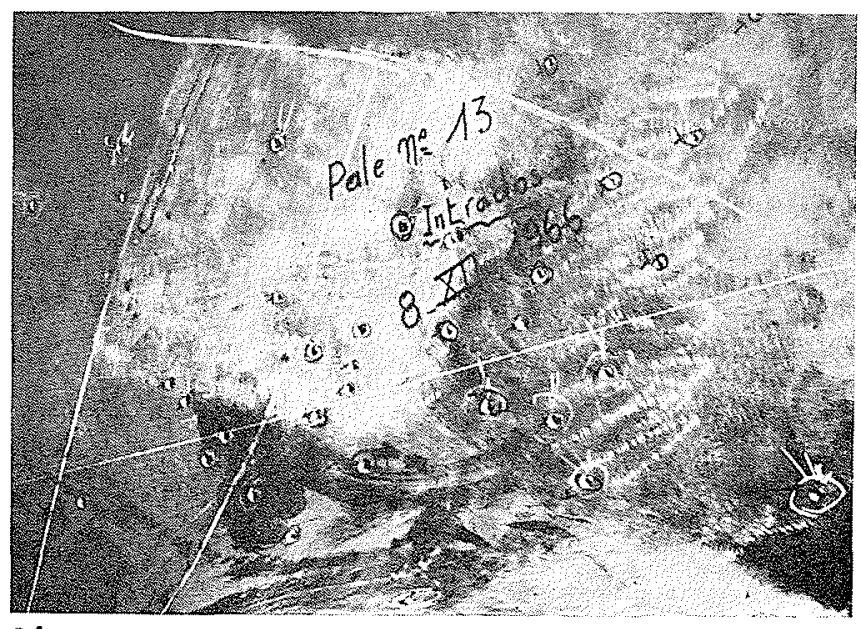


Les autres pièces métalliques, en acier ordinaire ou inoxydable, n'ont pas particulièrement souffert, car la peinture de protection, avec sa couche antifouling, était neuve et jouait pleinement son rôle; bien entendu, si de telles conditions d'immersion se produisaient maintenant, il n'en serait plus de même pour ces pièces, car la peinture est partie en beaucoup d'endroits et se trouve remplacée par le dépôt calcomagnésien dû à la protection cathodique.

Sur les autres groupes, les pales en bronze d'aluminium se sont toujours parfaitement comportées.

\subsection{Pendant la deuxième période d'immersion, avec rota- tion et protection cathodique,}

les pièces non revêtues de peinture de protection se sont, cette fois, très bien comportées, notamment les pales en acier martensitique 17/4, sans aucune apparition de dépôts ou de piqûres, sauf pour les tuyauteries dont nous parlerons plus loin. Sur les zones non protégées des pièces en acier ordinaire, et même sur des pales en bronze, il s'est alors produit un dépôt calco-magnésien.

Actuellement, après 40000 à 50000 heures d'immersion $\left({ }^{a}\right)$ et 25000 à 30000 heures de fonctionnement en rotation, le comportement de ces pièces est toujours excellent.

Il faut notamment signaler qu'au recouvrement de surfaces métalliques, comme par exemple «directrice - flasque supérieur ou inférieur $»$, aucune corrosion n’a été décelée.

Par contre, une corrosion intense s'est développée sur la pièce d'usure du joint Simrit, en amont du joint d'arbre; cette pièce, en acier austenitique 20/10/3, a été transformée en véritable «fromage de gruyère»; il faut noter que cette pièce ne se trouve pas dans le champ de la protection cathodique, ce qui explique mieux ce phénomène.

\subsection{Pendant ces deux périodes de mise en ecru des groupes.}

on a donc pu noter les points suivants:

- le bon comportement des pièces en bronze d'aluminium, avec ou sans protection cathodique, compte tenu de la propriété « antifouling 》 évidente $(b)$;

- la nécessité, pratiquement absolue, d'une protection cathodique pour les aciers ordinaires, la peinture n'assurant plus une protection suffisante après plusieurs mois d'immersion;

- la nécessité de cette protection pour les aciers martensitiques $17 / 4$, pour éviter le dépôt des algues et des coquillages, entraînant des corrosions localisées en eau stagnante.

(') Au début du mois d'octobre 1972

${ }^{(b)}$ Les grilles, en bronze d'aluminium, des tuyauteries de vidange $\varnothing 350$ des conduits hydrauliques des groupes, avaient été peinte avec de l'Araldite; les coquillages se sont déposés sur cette peinture et ont obstrués 60 à $80 \%$ de l'ouverture; il a fallu enlever la peinture pour mettre le bronze d'Alu à nu et arrêter ce dépôt de coquillages.

\section{4. - Comportement des métaux des vannes et des écluses}

Pour les vannes du barrage et les portes d'écluse, on retrouve également deux périodes de fonctionnement comme précédemment :

- une première période, jusqu'en mars 1968 pour les vannes, et mars 1970 pour les portes d'écluse, sans protection cathodique;

- une 'deuxième période, après ces dates, où la protection a été installée.

\subsection{Comportement des vannes de barrage.}

- Pendant la première période, sans protection cathodique, on a observé un bon comportement des rails de roulement en acier martensitique $17 / 4$, mais une corrosion très importante des fusées en acier ordinaire des galets de roulement.

Les galets sont en acier $17 / 4$, comme les chemins de roulement, et on a isolé les fusées des têtiers du tablier en acier ordinaire avec des joints isolants. Ce bimétalisme a été la cause de cette intense corrosion.

Le tablier, en acier ordinaire, qui était peint, n'a pas souffert tant que la peinture était en état; mais il aurait fallu la refaire assez fréquemment pour éviter de notables dégradations.

- Pendant la deuxième période, la protection cathodique des vannes a permis d'empêcher la corrosion des tabliers, malgré la dégradation de la peinture; toutefois, au début, les fusées restant isolées du tablier, elles n'étaient pas protégées par la protection cathodique. On a alors court-circuité l'isolant pour les relier au tablier; le courant de la protection cathodique a beaucoup augmenté, car on protège également des fers à béton, mais la corrosion des fusées a été arrêtée.

\subsection{Comportement des écluses.}

Pendant la première période, sans protection cathodique, de nombreuses corrosions ont été observées:

- Corrosion intense des membrures et montants tubulaires, en acier ordinaire, des petites vannes des aqueducs, allant jusqu'au percement.

- Corrosion des grilles d'aqueducs en acier ordinaire peint avec de la peinture vinylique.

- Les portes d'écluse sont verticales et le joint d'étanchéité des pivots était constitué d'un anneau de mercure dans lequel trempait une paroi cylindrique en acier ordinaire; cette tôle a littéralement fondu par effet d'électrolyse. On a supprimé le mercure et mis un compound très épais, ce qui a réglé ce problème.

Pendant la deuxième période, la protection cathodique, à anodes consommables, a réduit la corrosion des vannes d'aqueduc, sauf au-dessous des membrures où les coquillages continuent à s'accumuler; on va mettre une anode à la partie inférieure pour protéger cette zone.

Les grilles d'aqueduc, n'étant pas intéressées par la protection cathodique, ont continué à se corroder malgré la peinture. On va les relier à la masse des portes pour les protéger.

La corrosion des portes, elle, a été stoppée dans les endroits où la peinture était partie. 


\section{5. - Problème particulier de la visserie}

Il existe beaucoup de visserie, en acier ordinaire cadmiébichromoté, peinte sur les vannes et portes d'écluses notamment. Quand elle est bien peinte, et avec la protection cathodique, son comportement est satisfaisant. En l'absence de protection cathodique, il faut bien surveiller la peinture pour qu'elle soit toujours en état.

Il y a peu de visserie en acier inoxydable; à cause de la corrosion par aération différentielle, elle tient généralement assez mal quand il y a de l'eau dans les filets.

La visserie en bronze d'aluminium tient mieux que la précédente et on l'utilise généralement sur des pièces inox.

MM. Weill-Couly et Arnauld, dans une communication aux Journées d'Automne de la Métallurgie, montrent que la tenue en eau de mer du bronze d'aluminium est satisfaisante si les conditions suivantes sont remplies simultanément:

$$
\begin{aligned}
& \text { - pourcentage d'Al }<8,5 \%+\frac{\% \text { de Ni }}{2} \\
& \text { - pourcentage de Ni }>1,5 \%
\end{aligned}
$$

Il semble également que le pourcentage de $\mathrm{Mn}$ doit être inférieur à $2 \%$.

Ces conditions ont été mises en évidence après analyse de nombreux boulons corrodés et non corrodés en eau de mer.

\section{6. - Comportement des tuyauteries}

\subsection{Tuyauleries en acier inoxydable à l'intérieur des groupes.}

Une attaque des tuyauteries en acier austénitique $18 / 8$ des soupapes d'entrée d'air, entre la roue et les directrices des turbines, s'est développée au voisinage de soudures et est allée jusqu'au perçage.

Il semble que cette corrosion soit due à une corrosion intergranulaire, résultant d'une précipitation de carbure de chrome lors de l'élévation de la température du métal au moment du soudage.

Devant ce phénomène, on a alors simplement supprimé les tuyauteries inutiles (canalisations d'aération), ce qui est le remède le plus simple! et les autres ont été remplacées par des tuyauteries en matière plastique résistante.

D'autres attaques de corrosion ont été 'décelées sur les tuyauteries d'amenée d'eau d'arrosage de joints d'étanchéité, au voisinage du raccordement à la boîte à eau sur les raccords filetés.

Sur les brides en acier inoxydable, on a également observé de la corrosion, par aération différentielle, quand la fuite des joints conduisait à avoir de l'eau stagnante entre les brides.

\subsection{Tuyauteries en bronze d'aluminium.}

On a observé de nombreuses corrosions locales allant jusqu'au percement; ce fut le cas de tuyauteries de $\varnothing 150 \mathrm{~mm}$ d'aspiration des pompes distribuant l'eau de mer pour l'arrosage des joints d'arbre, et des tuyauteries de vidange des conduits des groupes.

Ces tuyauteries avaient $3 \mathrm{~mm}$ d'épaisseur environ et $2 \%$ de $\mathrm{Ni}, 2 \% \mathrm{Fe}, 1,5 \% \mathrm{Mn}$.

Il semble que la perforation soit due à une désaluminisation au voisinage des soudures et, se situant dans les parties supérieures, là où il y a de l'eau stagnante. Une grande partie de ces tuyauteries ont été remplacées par des tuyaux en matière plastique; pour celles qui restent, on va essayer un traitement thermique après soudage, consistant à chauffer la zone de soudure à $850^{\circ} \mathrm{C}$ pendant 20 minutes, puis à revenir à $600^{\circ} \mathrm{C}$ à raison de $50^{\circ} \mathrm{C}$ par heure, et ensuite de laisser refroidir à l'air.

\section{7. - Conclusion}

En conclusion, on peut dire que tous les problèmes de corrosion sont résolus avec la protection cathodique; en effet, sans cette protection, il serait très difficile de protéger les pièces en acier ordinaire d'une façon efficace et même certaines pièces en acier noble, comme les aciers austénitiques et martensitiques. 


\title{
DISCUSSION DES COMMUNICATIONS
}

\author{
M. FARAL: \\ LES DIFFÉRENTS TYPES DE PROTECTION CONTRE LA CORROSION MIS EN GUVRE A L'USINE \\ MARÉMOTRICE DE LA RANCE
}

M. LEBORGNE :

COMPORTEMENT DES MÉTAUX A L'USINE MARÉMOTRICE DE LA RANCE

Présidenl: M. P. CaBaniols

M. WeIL-Couly (F.F.A.H.R.) apporte les précisions suivantes sur sa formule citée dans le mémoire de M. Leborgne:

La formule:

$$
\text { Al } \% \leqslant 8,5 \%+\frac{\mathrm{N}_{1} \%}{2}
$$

conditionne vraisemblablement la possibilité de transformation de la phase $\beta$ suivant le schéma:

$$
\beta \longrightarrow \alpha+K_{I I}
$$

Cette décomposition doit être au moins partielle pour qu'il y ait protection contre la corrosion sélective.

Une telle dissociation de la phase $\beta$ qui n'est possible que si la formule ci-dessus est vériftée, n'est, en fait, réalisée que si la vitesse de refroidissement entre $850^{\circ} \mathrm{C}$ et $650^{\circ} \mathrm{C}$ est suffisamment lente. Une vitesse de 50 à $100^{\circ} / \mathrm{h}$ conduit à une transformation à peu près totale. Dans le cas d'une soudure sur une tôle mince, il peut y avoir stabilisation de la phase $\beta$ dans la zone affectée thermiquement du fait d'une vitesse de refroidissement trop rapide et ainsi risque de corrosion sélective de cette phase $\beta$; doù le traitement thermique cité dans la communication de M. Leborgne.

M. Auroy pose les deux questions ci-après:

$1^{\circ}$ La ou les couches antifouling sont-elles indispensables dans la protection de surface?

$2^{*}$ Y a-t-jl une relation entre l'établissement d'une protection cathodique et l'application d'un revêtement de surface, notamment dans le but de réaliser une économie éventuelle sur cette dernière, par exemple sur son épaisseur?

M. Faral répond:

Lexistence de couches de finition anti-fouling, indépendamment du fait qu'elles permettent de conserver un état de surface hydrauliquement plus satisfaisant, ce qui est un autre problème, facilitent en général la tâche des couches de protection proprement dites, en supprimant l'agressivité supplémentaire apportée par un milieu vivant. Leur nécessité, lorsqu'il existe par ailleurs une protection cathodique, peat se discuter, l'action anti-fouling d'une telle protection étant d'ailleurs controversée.

11 est vrai que dans 19 mesure où une protection cathodique est prévue, on peut être moins exigeant sur les caractéristiques du revêtement de surface appliqué à lorigine, tant en ce qui concerne sa perfection en tous points que sa durée. La présence d'un revêtement permet un démarrage beaucoup plus aisé de la protection cathodique, qui devra être alors dimensionnée en fonction du taux de disparition du revêtement au cours du temps.

M. Remenieras pose la question ci-après:

Certains projets de pisciculture dans la zone maritime de quelques «abers» bretons prévoient la construction de barrages - noyés à la haute mer - surmontés sur plusieurs mètres de hauteur, d'un écran vertical en toile métallique. On a proposé de constituer ces toiles de fils d'acier - inoxydable ou non - revêtus de matière plastique. A la lumière de l'expérience de la Rance, quelle est la solution qui résistera le mieux à la corrosion?

M. LAMBERT (GAZ DE FRANCE) répond:

Je ne pense pas que l'utilisation de filets en acier inoxydable recouvert d'un revêtement plastique puisse être considéré comme optimale.

En effet, l'acier inoxydable n'est incorrodable qu'en milieu oxydant ou oxygéné, cette incorrodabilité résultant d'une couche superficielle de passivité. Il nous paraît donc logique d'utiliser:
- soit l'acier inoxydable seul sous réserve que la qualité en soit garantic pour tenir dans le milieu considéré,

- soit d'utiliser un acier au carbone à 50-60 hbars, revêtu do polyamide, une protection cathodique peu onéreuse assurant la stabilité de l'acier ordinaire au droit des défauts du revêtement. De toute façon, la protection contre la corrosion par aération différentielle au croisement des fils en acier inoxydable de la toile posera des problèmes difficiles.

Depuis Ia construction de l'usine de la Rance, la technique des peintures a fait des progrès, observe $M$. Duhoux. Profitant do l'expérience acquise, quel type de peinture utiliserait-on aujourd'hui pour protéger les «groupes» avant leur mise en service?

D'après les rapports d'E.D.F. et aussi certains mémoires japonais, dit M. Merstre, il suffirait - là où il n'y a pas nécessité de laisser lacier nu - d'utiliser de l'acier ordinaire, de le protéger avec une bonne peinture et de réaliser une excellente protection cathodique; même si la protection cathodique se trouvait accidentellement hors service pendant plusieurs jours - peutêtre même quelques semaines - le dépôt magnésique dont on a parlé suffirait à assurer une protection temporaire des surfaces métalliques.

Lors des essais du groupe expérimental de Saint-Malo, M. LeborGNE ayant été voir une porte d'écluse des Ponts-et-Chaussées à Saint-Malo ét les circuits d'eau de mer de l'usine thermique souterraine de Porzic, indique avoir été très inquiet par l'intensité que prenaient les phénomènes de corrosion. Heureusement la protection cathodique a été mise au point à l'usine marémotrice de la Rance et cela a été un succès éclatant; il faut bien dire que sans cette protection, l'exploitation de l'usine serait très difficile.

La durée de l'efficacité des peintures anti-fouling est estimée à un à quatre ans selon la vitesse du courant. Cette peinture est-elle renouvelée ou seulement retouchée lorśs des révisions annuelles des groupes de la Rance, demande M. Meystre?

La peinture anti-fouling tient deux à trois ans, répond M. MarolLEAU mais, confiant dans la protection cathodique, on ne procède pas à sa réfection au bout de ce délai; on attend le moment de la réfection de l'ensemble des peintures (1): les peintures anti-fouling ou anti-salissure permettent surtout de limiter les dépôts d'algues et de coquillages qui peuvent perforer la pellicule sous-jacente de peinture de base.

M. Meystre se pose la question suivante:

Que se passerait-il si lon ne renouvelait plus cette peinture? Probablement rien, grâce au dépôt calco-magnésien dont on vient de parler. Tout au plus, peut-on dire qu'à ce moment - donc sans protection cathodique et sans peinture anti-salissure - les organismes vivants pourraient se fixer si, de plus le groupe devait être en eau stagnante. Mais alors, si cela devait être gênant, il ne faudrait pas «attendre encore un certain temps» pour renouveler la peinture! C'est en fin de compte une question d'économie.

D'autre part, s'il semble démontré que la protection cathodique par courant imposé et les ultra-sons tuent les larves des mollusques, est-on aussi bien renseigné sur leur action sur les algues?

M. le Président clôt la discussion et déclare en conclusion:

Si nous sommes tous convaincus que le choix des matériaux et des peintures est important dans tous ces problèmes de corrosion, il semble bien, qu'en définitive, la sécurité est apportée par la protection cathodique.

(1) Il est fortement déconseillé de refaire une colache de peirture anti-fouling sans avoir repris la couche sous-jacente car si cette derniere n'est pis ctanche on créte des conples cuivre-fer. 